\title{
The combination of cantharidin and antiangiogenic therapeutics presents additive antitumor effects against pancreatic cancer
}

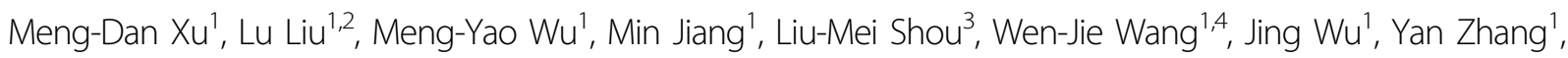
Fei-Ran Gong ${ }^{5}$, Kai Chen ${ }^{1}$, Min Tao ${ }^{1,6}$, Qiaoming Zhi ${ }^{7}$ and Wei Li ${ }^{1,6,8}$

\begin{abstract}
Cantharidin, one of the active components of mylabris, is believed to have antitumor activity. Cantharidin selectively inhibits protein phosphatase 2A (PP2A), which can repress multiple oncogenic kinases (ERK, JNK, PKC, and NF-KB). Researches in vitro have shown that cantharidin suppresses cell viability and metastasis in pancreatic cancer cells. This study aims to investigate the effects of cantharidin on pancreatic cancer xenografts in vivo. Xenograft models were established using cells stably expressing luciferase. Xenograft growth was evaluated by living imaging. Gene expression was determined using a microarray, real-time PCR, a RayBiotech antibody array, and the Milliplex assay. Surprisingly, cantharidin significantly accelerated xenograft growth. Living imaging showed a rapid distribution of Dluciferin in cantharidin-treated xenografts, suggesting a rich blood supply. Immunohistochemistry confirmed increased angiogenesis. Microarray and antibody array identified upregulated proangiogenic and downregulated antiangiogenic factors. The Milliplex assay suggested elevated secretion of IL-6, IL-8, TNF-a, and VEGF. Inhibitors of ERK, JNK, PKC, and NF-KB pathway attenuated the cantharidin-induced changes to proangiogenic gene expression. PKC pathwayinhibiting tamoxifen or antiangiogenic therapeutics, including Ginsenoside Rg3, bevacizumab, Apatinib, and Endostar, antagonized the proangiogenic effect of cantharidin or its derivatives. These regimens presented remarkable additive antitumor effects in vivo. Although cantharidin presents antitumor effects in vitro and has been applied in clinical practice, we revealed an unfavorable proangiogenic side effect. We recommend that the clinical application of cantharidin should be performed on the premise of antivascularization therapy.
\end{abstract}

\section{Introduction}

Pancreatic cancer is a malignant disease, the mortality of which almost parallels its incidence ${ }^{1}$. Compared with

Correspondence: Min Tao (taomin@suda.edu.cn) or

Qiaoming Zhi (strexboy@163.com) or Wei Li (liwei10@suda.edu.cn)

(dr_weili@163.com)

'Department of Oncology, the First Affiliated Hospital of Soochow University, 215006 Suzhou, China

2Department of Emergency, the First Affiliated Hospital of Soochow University, 215006 Suzhou, China

Full list of author information is available at the end of the article.

These authors contributed equally: Meng-Dan Xu, Lu Liu, Meng-Yao Wu, Min Jiang the steady increase in the survival rate of most cancers, little progress has been made in pancreatic cancers. More than $50 \%$ of patients suffering from this disease are diagnosed at advanced or distant stages and are refractory to conventional treatments ${ }^{2}$. It is estimated that the current 5 -year relative survival is $8 \%$ in the United States (2017) and continues to increase slightly (by $0.3 \%$ per year) in men $^{3}$. Therefore, new strategies are urgently required to overcome this malignant disease.

Cantharidin is one of the active ingredients of mylabris. It is believed to have antitumor effect and has been widely

\section{(c) The Author(s) 2018}

(c) (i) Open Access This article is licensed under a Creative Commons Attribution 4.0 International License, which permits use, sharing, adaptation, distribution and reproduction cc) in any medium or format, as long as you give appropriate credit to the original author(s) and the source, provide a link to the Creative Commons license, and indicate if changes were made. The images or other third party material in this article are included in the article's Creative Commons license, unless indicated otherwise in a credit line to the material. If material is not included in the article's Creative Commons license and your intended use is not permitted by statutory regulation or exceeds the permitted use, you will need to obtain permission directly from the copyright holder. To view a copy of this license, visit http://creativecommons.org/licenses/by/4.0/. 
used in China. Cantharidin selectively inhibits protein phosphatase 2A (PP2A), a repressor of several oncogenic kinase pathways, including extracellular signal-related kinase (ERK), c-Jun N-terminal kinase (JNK), protein kinase $\mathrm{C}(\mathrm{PKC})$, and nuclear factor kappa-light-chainenhancer of activated B cells (NF-kB), all of which play important roles in controlling cell cycle, apoptosis, and determining cell fate ${ }^{4}$. Therefore, it is contradictory that cantharidin, an inhibitor of cancer-repressing PP2A, should present an antitumor effect. Our previous studies demonstrated that sustained activation of the JNK and NF- $\mathrm{KB}$ pathways, induced by PP2A inhibition, was responsible for the growth inhibition of cantharidin, indicating that activation of these kinase pathways was not always facilitating cancer progress. Moreover, cantharidin inhibited migration, arrested the G2/M cell cycle transition, induced apoptosis, repressed invasion, and impaired the stemness of pancreatic cancer cells in vitro ${ }^{4-11}$. However, these antitumor effects of cantharidin have not been verified in pancreatic cancer in vivo. So this study aimed to investigate the effect of cantharidin on pancreatic cancer xenografts in vivo.

\section{Results}

Cantharidin accelerated the growth of pancreatic cancer in both subcutaneous and orthotopic xenografts

As shown in Fig. 1a, b, surprisingly, the mice in the cantharidin-treated group showed significant body weight loss and enlarged tumor volumes compared with the control group. Living imaging showed that cantharidin significantly accelerated the growth of pancreatic cancer subcutaneous xenografts, rather than inhibiting them (Fig. $1 \mathrm{c}-\mathrm{f})$. Moreover, we identified similar results in lung cancer and colorectal cancer (Supplementary Fig. 1). Methyl thiazolyl tetrazolium (MTT) assays showed that cantharidin exhibited an inhibitory effect on the growth of both NCI-H292 lung cancer cells (Supplementary Fig. 1A) and LoVo colorectal cancer cells (Supplementary Fig. 1B) in vitro as well. However, in in vivo studies, cantharidin promoted the growth of NCI-H292 and LoVo xenografts (Supplementary Fig. 1C-F), suggesting that the progrowth effect of cantharidin was independent of cancer type. To further confirm this confusing result, we established orthotopic xenograft models and found consistent results (Fig. $1 \mathrm{~g}-\mathrm{k}$ ). Interestingly, by scanning the process using living imaging (Fig. 1l, m), we noticed that the bioluminescence peak value of the cantharidin-treated subjects emerged earlier than the control group. In addition, the bioluminescence of the cantharidin group also decayed faster, indicating a rapid distribution of Dluciferin. This phenomenon suggested that cantharidintreated xenografts might have a rich blood supply, which led us to speculate that cantharidin might promote angiogenesis in vivo.

\section{Cantharidin promoted angiogenesis of a pancreatic cancer model}

To verify the hypothesis that cantharidin might promote angiogenesis, expression of CD34 was detected in cantharidin-treated pancreatic orthotopic xenograft specimens via immunohistochemistry. As shown in Fig. 2a, vascular endothelial cells were stained with CD34 antibody by immunohistochemistry. According to microvessel density (MVD) levels, we found that treatment with cantharidin promoted angiogenesis significantly compared with the control subjects. Similar results were obtained in xenografts of lung cancer (NCI-H292 cells) and colorectal cancer (LoVo cells), shown in Supplementary Fig. 2a, b, suggesting that the proangiogenic effect of cantharidin was independent of cancer type.

Angiogenesis is executed via activating multiply kinase pathways, including JNK, ERK, PKC, and NF- $\mathrm{KB}^{12-15}$. All these pathways share the same negative regulator, PP2A. Previously, we have proved that cantharidin could activate the pathways of ERK, JNK, PKC, and NF- $\mathrm{kB}$ through inhibition of $\mathrm{PP}^{2} \mathrm{~A}^{4-7,11}$. Therefore, the proangiogenic effect of cantharidin could be related to PP2A inhibition. To verify this hypothesis, we assessed the secretion and expression of proangiogenic factors upon treatment with cantharidin and okadaic acid (OA), a classic PP2A inhibitor.

First, we used milliplex assays to measure the levels of proangiogenic interleukin (IL)-6, IL-8, tumor necrosis factor- $\alpha$ (TNF- $\alpha$ ), and vascular endothelial growth factor (VEGF) in culture medium of PANC-1 cells upon treatment with cantharidin or OA. As expected, both cantharidin and $\mathrm{OA}$ time-dependently promoted the secretion of IL-6, IL-8, TNF- $\alpha$, and VEGF (Fig. 2b).

Then we analyzed microarray data ${ }^{5}$ to determine the mRNA expression levels of angiogenesis-related genes upon treated with cantharidin or OA in PANC-1 cells. The microarray data are available from Gene Expression Omnibus (GEO) database, the accession code of which is GSE114288. As presented in Fig. 2c, both cantharidin and OA upregulated the expression levels of multiple proangiogenic genes, including TIMP1, CXCL1/GRO- $\alpha$, CXCL2/GRO- $\beta$, IL-8/CXCL8, PLAUR/UPAR, RUNX1, PGF/PIGF-2, VEGF/VEGFA, RHOB, BTG1, CD55, SPHK1, EFNB2, IL12A, CXCL5/ENA78/LIX, IL-10, BAI1, CXCL3/GRO- $\gamma$, TNF/TNF- $\alpha$, GM-CSF/CSF2, F3, KITLG, TIE1, CSF3, SERPINC1, IL-1 $\alpha, C X C L 14$, and FGF1. The expression of antiangiogenic COL18A1/Endostatin was downregulated following cantharidin and OA treatments.

Furthermore, we assessed the protein levels of the angiogenesis-related genes after cantharidin or OA treatment using RayBio antibody arrays. Both cantharidin and $\mathrm{OA}$ upregulated the levels of provascularizing Angiogenin, epidermal growth factor EGF, GRO, IL-6, IL8, LEPTIN, platelet-derived growth factor-BB, placenta 


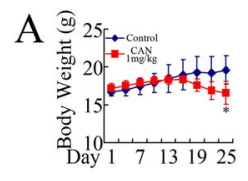

B

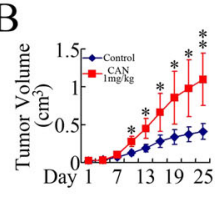

$\mathrm{G}$

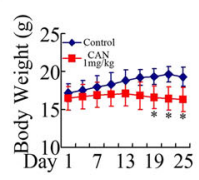

C

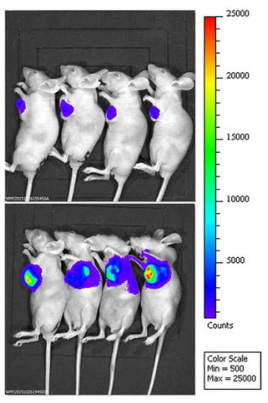

$\mathrm{H}$

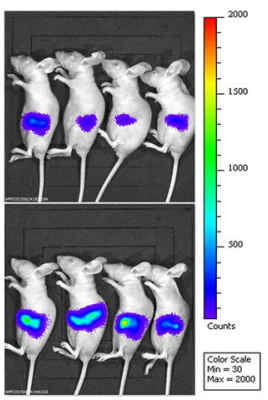

D E

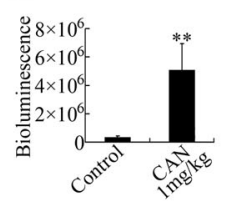

E

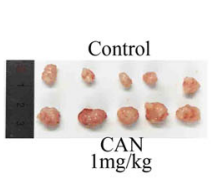

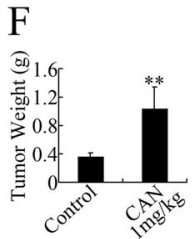

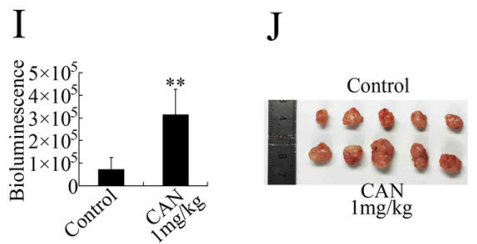

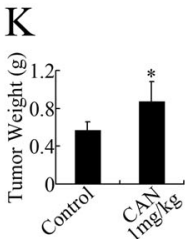

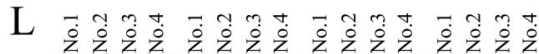

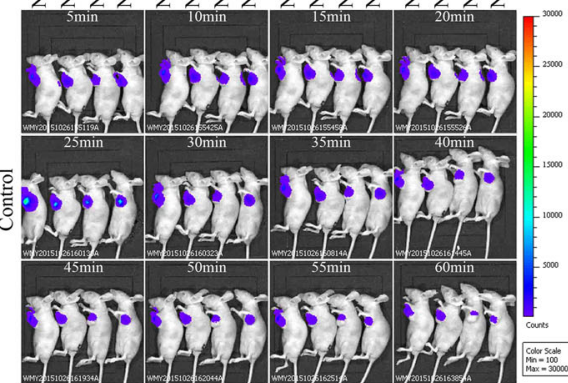

M
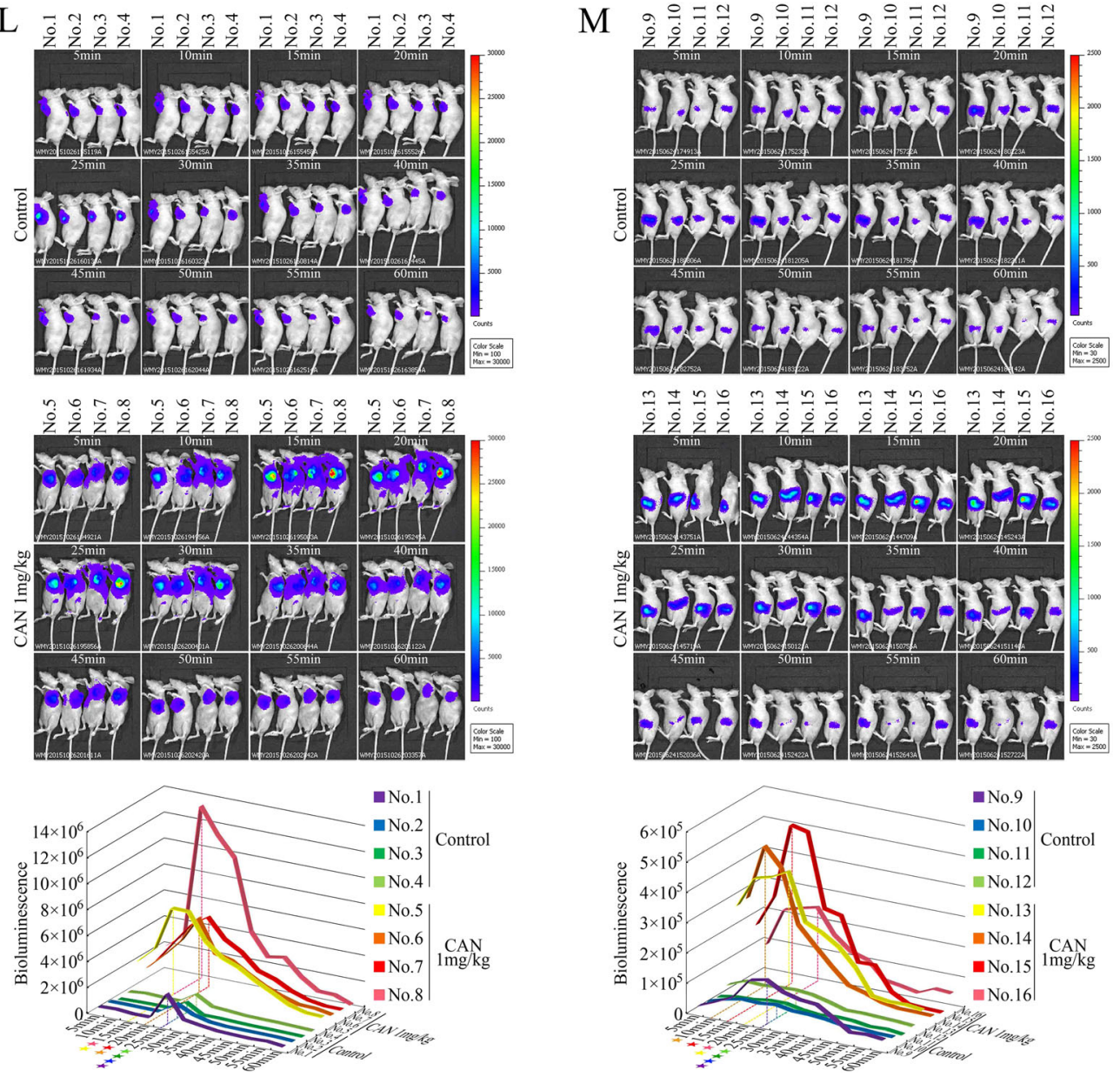

Fig. 1 (See legend on next page.) 
(see figure on previous page)

Fig. 1 Cantharidin accelerated the growth of pancreatic cancer in both subcutaneous and orthotopic xenografts. PANC-1 cells were used in both subcutaneous and orthotopic pancreatic xenograft models. a, b Body weight (a) and tumor volume (b) evaluations of the cantharidin (CAN) group vs. the control group in subcutaneous xenografts. c, $\mathbf{d}$ Representative in vivo bioluminescent images (c) and bioluminescence analysis (d) of pancreatic cancer subcutaneous xenografts. $\mathbf{e}, \mathbf{f}$ Photographs of subcutaneous xenograft (e) and tumor weight (f) in the CAN vs. the control group. $\mathbf{g}$ Body weight of the CAN vs. the control group in orthotopic xenografts. $\mathbf{h}$, i Representative bioluminescent images (h) and bioluminescence analysis (i) of pancreatic cancer orthotopic xenografts. j, k Photographs of orthotopic xenograft (j) and tumor weight (k) in the CAN vs. the control group. I, $\mathbf{m}$ The bioluminescent images showing the process of bioluminescence peak emergence and decay in the CAN vs. the control group in subcutaneous (I) and orthotopic xenografts $(\mathbf{m}) .{ }^{*} P<0.05$ and ${ }^{*} P<0.01$, significant differences vs. the control group

growth factor, VEGFA, granulocyte macrophages colonystimulating factor (GM-CSF), IL-1 $\alpha$, matrix metalloproteinase (MMP)-1, and urokinase-type plasminogen activator receptor (uPAR) (Fig. 2d). By cross-comparing the microarray datasets, we found that both cantharidin and OA could increase expression levels of $I L-8 / C X C L 8$, CXCL1/GRO- $\alpha, \quad V E G F / V E G F A, \quad G M-C S F / C S F 2$, and $P L A U R / U P A R$ at both mRNA and protein levels. Therefore, the proangiogenic effect of cantharidin and OA may be executed through mechanisms involving upregulating multiple angiogenesis-related genes.

ERK, JNK, PKC, and NF-KB kinase pathways participated in cantharidin-induced upregulation of proangiogenic genes

Cantharidin could activate ERK, JNK, PKC, and NF- $\kappa B$ pathways by inhibiting PP2A ${ }^{4-7,11}$; therefore, we discussed whether these kinase pathways participated in the upregulation process of proangiogenic genes upon treatment with cantharidin.

PANC-1 cells were pretreated with the ERK inhibitor PD98059, the JNK inhibitor SP600125, the NF-KB inhibitor EF-24, and the PKC inhibitor GF109203X, separately, followed by cantharidin treatment. We then detected the expression of the proangiogenic genes that were upregulated by cantharidin using real-time PCR. Finally, we discovered that inhibitors of ERK, JNK, PKC, and/or NF- $\mathrm{KB}$ attenuated the cantharidin-induced remodeling of proangiogenic gene expression profiles significantly (Fig. 3), suggesting that all these pathways contribute to the provascularizing effect of cantharidin. In addition, inhibitors of NF- $\mathrm{KB}$ and PKC pathways were involved in the upregulation of most of the target genes (Table 2), implying that these two kinase pathways might be the key pathways responsible for the upregulation of proangiogenic genes upon treatment with cantharidin.

\section{Combination of cantharidin and Ginsenoside Rg3 or tamoxifen (TAM) presented additive anticancer effects}

Cantharidin presented multiple antitumor effects in vitro; therefore, we speculated that cantharidin might also be able to fulfill its anticancer capacity in vivo as long as the proangiogenic side effect was abolished. Based on this hypothesis, we investigated whether the combination of cantharidin and antiangiogenic therapeutics could present additive anticancer effects in vivo. Ginsenoside $\mathrm{Rg} 3$ is one of the active ingredients of ginseng, and it can suppress tumor angiogenesis by impairing the biological activity of endothelial progenitor cells and attenuating VEGF-dependent p38/ERK signaling and Akt/endothelial nitric oxide synthase signaling in vitro ${ }^{16,17}$. Clinical studies have proven that Rg3 exhibited a good safety record and few side effects ${ }^{18,19}$. TAM is a non-steroidal antiestrogen drug frequently used in endocrine therapy and has been proven to be an inhibitor of the PKC pathway ${ }^{11}$, which could be the main pathway participating in the proangiogenic effect of cantharidin (Fig. 3). We previously showed that TAM could repress the growth of pancreatic cancer cells by inhibiting the PKC pathway ${ }^{11}$. Since both $\mathrm{Rg} 3$ and TAM present satisfactory tolerance in clinical practice and can inhibit pathways participating in proangiogenic effect of cantharidin, we investigated whether $\operatorname{Rg} 3$ and TAM could antagonize the growthpromoting effect of cantharidin in vivo.

As the cytotoxicity of Rg3 against pancreatic cancer cells had not been verified, MTT assays were first performed in vitro (Fig. 4a). As expected, Rg3 inhibits PANC1 cell proliferation in both dose- and time-dependent manners. We then performed RNA-seq to obtain the gene expression profiles after treatment with Rg3 or TAM. As shown in Fig. 4b, although showing different patterns, both Rg3 and TAM repressed the expression of multiple angiogenesis-related genes. Moreover, both Rg3 and TAM reversed the growth-promoting effect of cantharidin and presented additive anticancer effects in combination with cantharidin (Fig. 4c-j).

\section{Antiangiogenic therapeutics antagonized the proangiogenic effect of cantharidin in vivo}

We then investigated whether specific antiangiogenic therapeutics could be more effective to constitute regimens with cantharidin. Three antiangiogenic therapeutics were used (Fig. 5a), including bevacizumab (a partially humanized monoclonal antibody targeting VEGF), Apatinib (an antiangiogenic agent targeting the intracellular ATP-binding site of VEGF receptor 2), and Endostar (a recombinant human endostatin). 


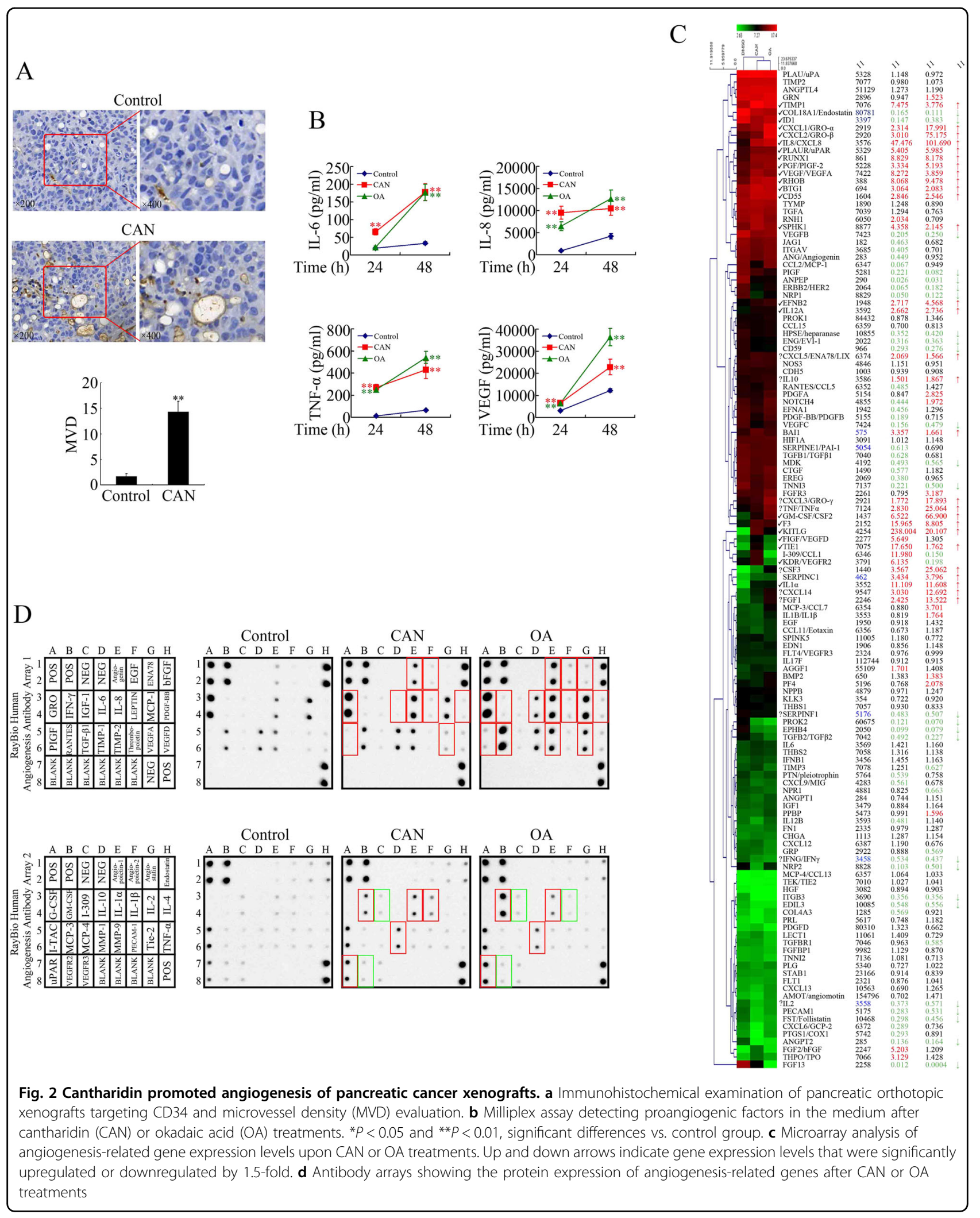


A

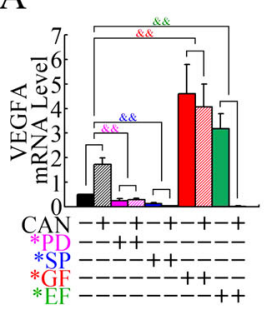

F

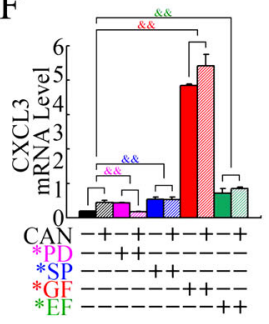

K

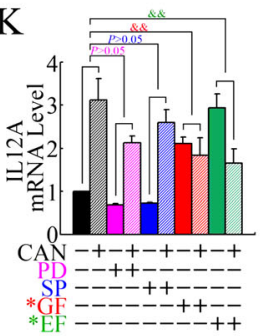

P

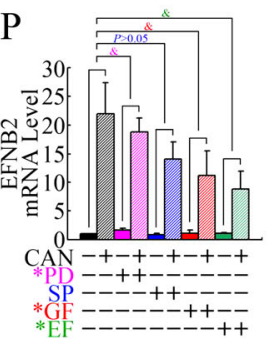

U

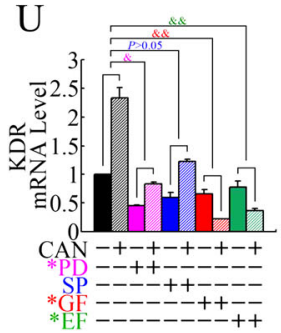

B

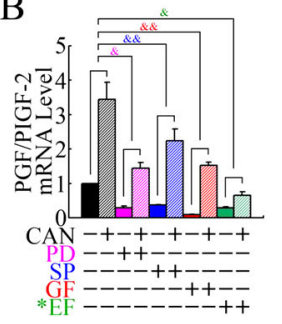

G

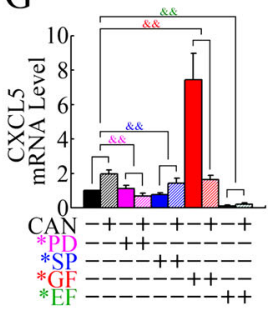

L

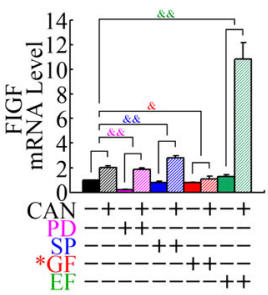

Q

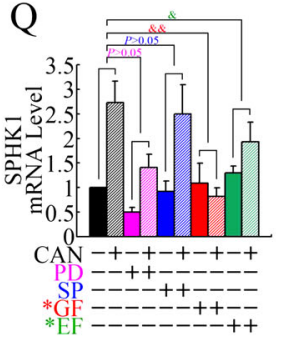

$\mathrm{V}$

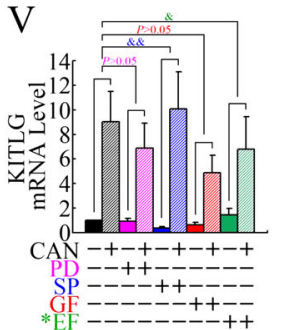

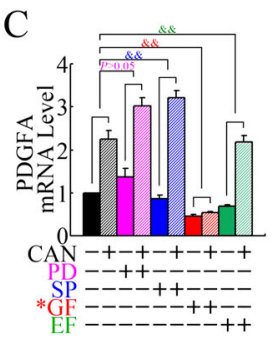

D

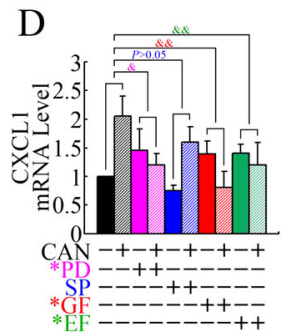

H

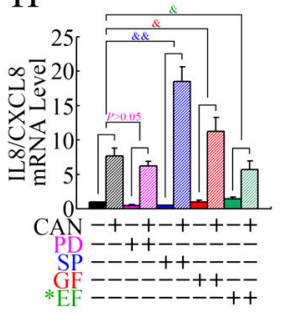

I
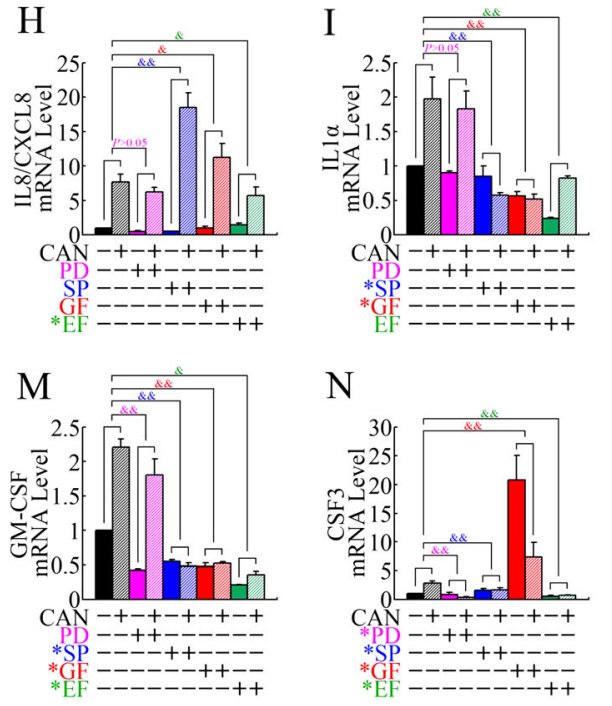

R
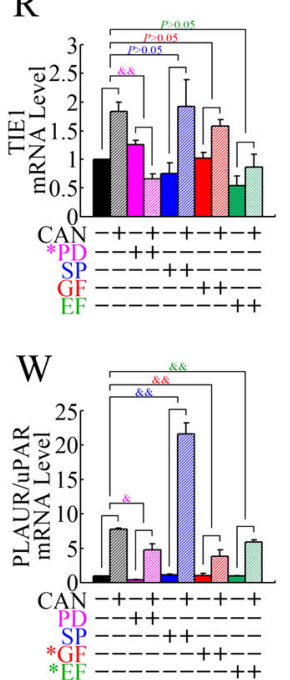

$\mathrm{N}$

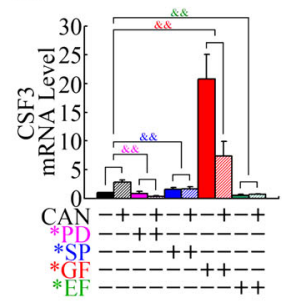

S

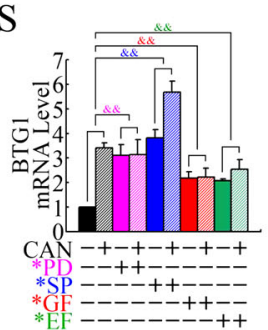

X

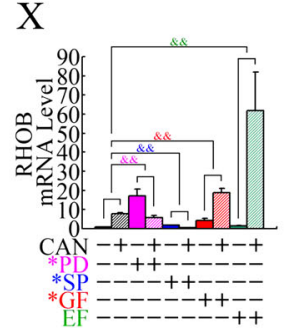

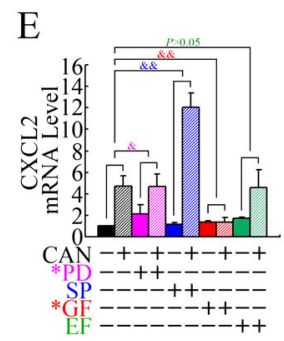

J

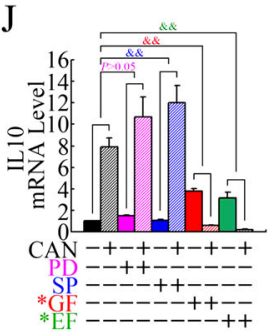

O

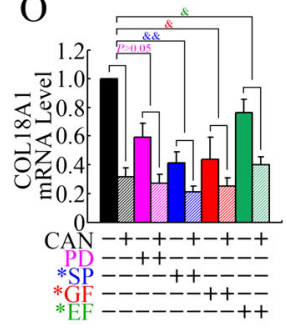

T
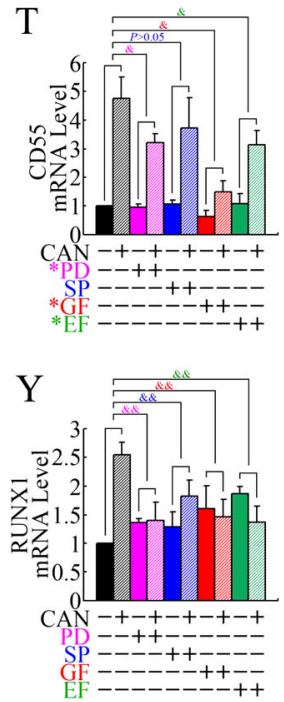

Fig. 3 ERK, JNK, NF-KB, and PKC kinase pathways contributed to cantharidin-induced upregulation of proangiogenic genes. a-y Real-time PCR determination of the mRNA levels of angiogenesis-related genes, including VEGFA (a), PGF/PIGF-2 (b), PDGFA (c), CXCL1 (d), CXCL2 (e), CXCL3 (f),

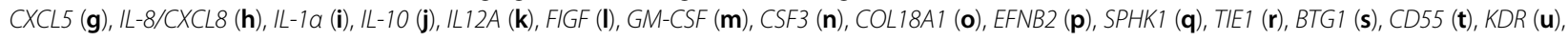
KITLG (v), PLAUR/UPAR (w), RHOB (x), and RUNX1 (y), in PANC-1 cells after treatment with cantharidin (CAN) combined with ERK pathway inhibitor PD98059 (PD), JNK inhibitor SP600125 (SP), PKC inhibitor GF109203X (GF), or NF-KB inhibitor EF-24 (EF). ${ }^{\&} P<0.05$ and ${ }^{\&} \&<0.01$, significant differences between fold changes. Asterisk $\left(^{*}\right)$ indicates that the inhibitors attenuated the CAN-induced upregulation of the target genes

As shown in Fig. 5b-e, when combined with bevacizumab, Apatinib, and/or Endostar, cantharidin repressed the growth of pancreatic cancer subcutaneous xenografts remarkably, indicating that cantharidin could play an effective anticancer role in vivo, as long as antiangiogenic therapeutics were applied. Notably, the 
Table 2 Summary of kinase pathways inhibitors attenuating the CAN-induced upregulation of proangiogenic genes

\begin{tabular}{|c|c|c|c|c|}
\hline \multirow[t]{2}{*}{ Genes } & \multicolumn{4}{|c|}{ Kinase pathway inhibitors } \\
\hline & PD98059 & SP600125 & GF109203X & EF-24 \\
\hline VEGFA & * & * & * & * \\
\hline PGF/PIGF-2 & & & & * \\
\hline PDGFA & & & * & \\
\hline CXCL1 & * & & * & * \\
\hline CXCL2 & * & & * & \\
\hline CXCL3 & * & * & * & * \\
\hline CXCL5 & * & * & * & * \\
\hline IL-8/CXCL8 & & & & * \\
\hline IL1a & & * & * & \\
\hline IL10 & & & * & * \\
\hline IL12A & & & * & * \\
\hline FIGF & & & * & \\
\hline GM-CSF & & * & * & * \\
\hline CSF3 & * & * & * & * \\
\hline COL18A1 & & * & * & * \\
\hline EFNB2 & * & & * & * \\
\hline SPHK1 & & & * & * \\
\hline TIE1 & * & & & \\
\hline BTG1 & $*$ & * & * & * \\
\hline CD55 & * & & * & * \\
\hline KDR & $*$ & & * & * \\
\hline KITLG & & & & * \\
\hline PLAUR/UPAR & & & * & * \\
\hline $\mathrm{RHOB}$ & * & * & * & \\
\hline RUNX1 & & & & \\
\hline
\end{tabular}

Asterisk ${ }^{*}$ ) indicates that the inhibitors attenuated the CAN-induced upregulation of the target genes

combination of bevacizumab, Endostar, and cantharidin almost abolished the enlargement of xenografts, suggesting that this regimen holds promise for future clinical applications.

Derivatives of cantharidin also presented in vivo growthpromoting effects that could be reversed by bevacizumab

Studies have shown that cantharidin has significant side effects, such as nephrotoxicity. Therefore, several derivatives of cantharidin have been developed for clinical practice. The most widely used derivatives are sodium cantharidinate (SCAN) and norcantharidin
(NCTD) (Fig. 6a). Considering the growth-promoting effect of cantharidin in vivo, it is necessary to evaluate whether the derivatives of cantharidin could also accelerate tumor growth. The cytotoxicities of SCAN and NCTD against pancreatic cancer cells were first verified in vitro using MTT assays. As expected, both SCAN and NCTD repressed the growth of pancreatic cancer cells in dose- and time-dependent manners, although the efficiencies of the derivatives were weaker than their precursor (Fig. 6a).

Consistent with cantharidin, both SCAN and NCTD promoted the growth of xenografts in vivo, which could be antagonized by bevacizumab (Fig. 6b-j). SCAN and NCTD are better tolerated than cantharidin; therefore, the combination of cantharidin derivatives and antiangiogenic therapeutics could represent more practical regimens.

Moreover, to better illustrate the roles played by cantharidin and its derivatives in solid tumors in vivo, we confirmed the antitumor effect of them in combination with antiangiogenic therapeutics in colorectal cancer subcutaneous xenografts models (Supplementary Figure 3). Compared with the control and single drug-treated groups, the tumors in the co-treated groups were much smaller, implying that cantharidin and its derivatives exhibited a significant antitumor effect in colorectal cancer when co-treated with antiangiogenic therapeutics.

\section{Discussion}

Cantharidin, as a traditional antitumor drug, has been widely used in clinical practice in China. The antitumor activity of cantharidin, involving inhibiting migration, triggering apoptosis, arresting $\mathrm{G} 2 / \mathrm{M}$ transition, and repressing invasion, has been explored in many in vitro studies $^{4-11}$. However, conflicting with these antitumor roles, subcutaneous and orthotopic xenograft models clearly illustrated that cantharidin could accelerate the growth of pancreatic, lung, and colorectal cancers in vivo. In addition, the emergence and decay of the bioluminescence peak value suggested that cantharidin-treated xenografts might have a rich blood supply. Higher $\mathrm{MVD}^{20}$ was detected in cantharidin-treated xenografts, confirming the proangiogenic effect of cantharidin in vivo.

Increasing evidence shows that aberrant angiogenesis plays an essential role in tumor progression in vivo ${ }^{19,21}$. Abundant angiogenesis regulatory factors and oncogene activation are critical for supporting tumorigenic angiogenesis phenotypes ${ }^{22}$. In the present study, a microarray and an angiogenesis antibody array suggested that cantharidin significantly upregulated the expression levels of proangiogenic IL-8, GRO, VEGF, GM-CSF, and uPAR at both mRNA and protein levels. Besides, the Milliplex assay exhibited that cantharidin promoted the secretion of proangiogenic VEGF, IL-6, IL-8, and TNF- $\alpha$. It is worth 


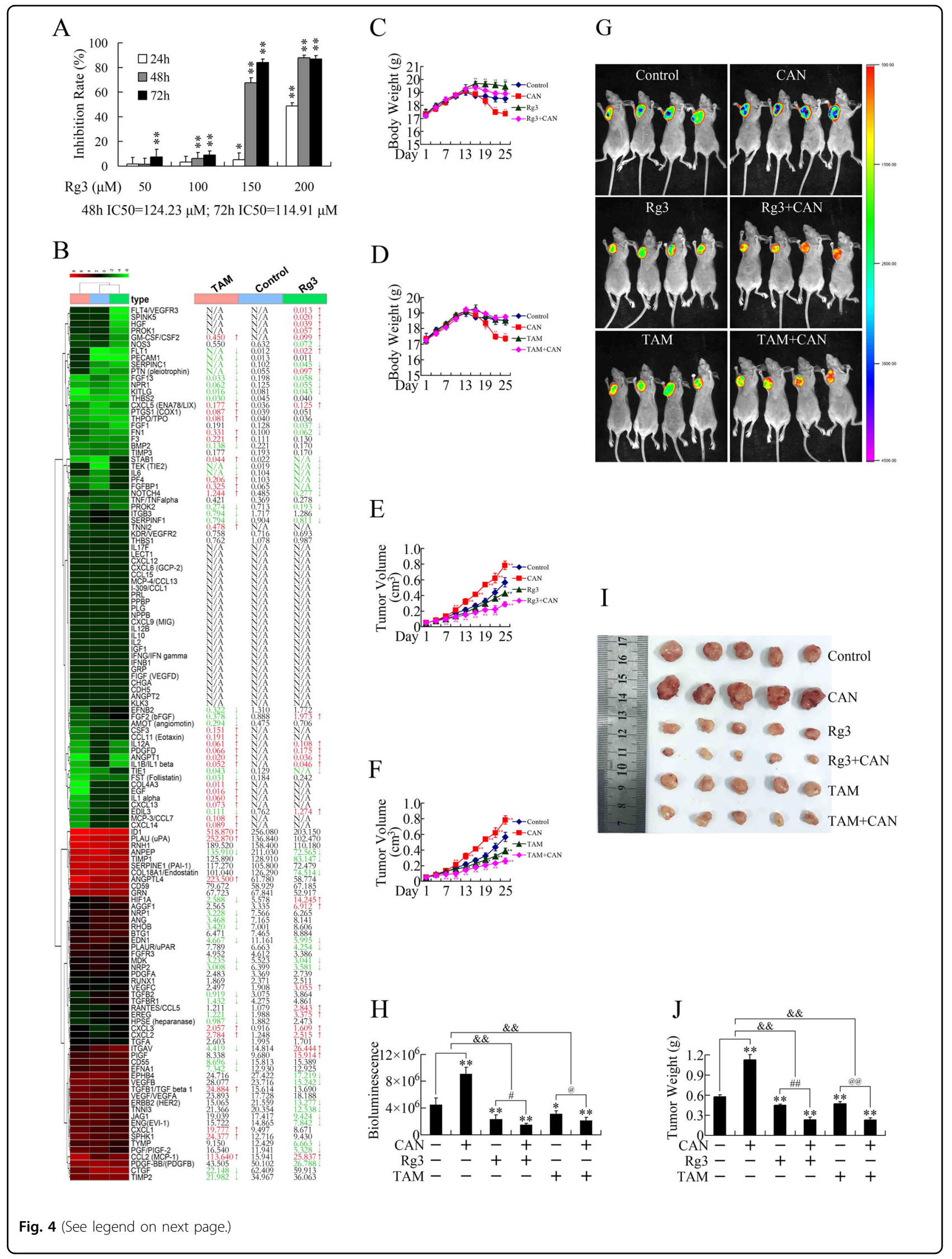




\begin{abstract}
(see figure on previous page)
Fig. 4 Both PKC-inhibiting tamoxifen and traditional medicine ingredient Rg3 attenuated the pro-growth effect of cantharidin in vivo. a Evaluation of the cytotoxicity of Rg3 in PANC-1 cells in vitro by MTT assay. Exposure to various concentrations of Rg3 inhibited PANC-1 cell growth in time- and dose-dependent manners. ${ }^{*} P<0.05$ and ${ }^{*} P<0.01$, significant differences vs. control group. $\mathbf{b}$ RNA-seq data of PANC-1 cells after treatment with tamoxifen (TAM) and Rg3. The up and down arrows indicate significantly upregulated or downregulated gene expression levels by 1.5 -fold. $\mathbf{c}, \mathbf{d}$ Body weight evaluations of subcutaneous xenograft models upon treatment with cantharidin and Rg3 (c) or TAM (d). e, f Tumor volume evaluations of subcutaneous xenografts after treated with cantharidin and Rg3 (e) or TAM (f). $\mathbf{g}, \mathbf{h}$ Representative in vivo bioluminescent images $(\mathbf{g})$ and bioluminescence analysis (h) showing the effects of cantharidin combined with Rg3 or TAM on pancreatic cancer subcutaneous xenografts. $\mathbf{i}$, $\mathbf{j}$ Photographs of subcutaneous cancer xenografts (i) and tumor weight evaluation (j) of subjects treated by cantharidin combined with Rg3 or TAM. ${ }^{*} P<0.05$ and ${ }^{* *} P<0.01$, significant differences vs. control group. ${ }^{\#} P<0.05,{ }^{\# \#} P<0.01,{ }^{@} P<0.05$, ${ }^{\circledR} P<0.01$, and ${ }^{\& \&} P<0.01$, significant differences between fold changes
\end{abstract}

noting that cantharidin increased VEGF and IL-8 not only at the mRNA level but also at the protein and secretion levels. VEGF exerts a crucial role in promoting of vascular endothelial cell growth and is vital for the process of angiogenesis ${ }^{23}$. IL-8 is a pro-inflammatory cytokine that acts as a chemotactic agent. Besides, it is also a powerful angiogenic factor ${ }^{24}$, which contributes to the distant metastasis of cancer ${ }^{25,26}$. Therefore, VEGF and IL-8 may exert a major role in the proangiogenic effect of cantharidin.

Cantharidin is a potent and selective inhibitor of PP2A, a repressor of several oncogenic kinase pathways, including ERK, JNK, PKC, and NF- $\mathrm{KB}$. Inhibition of PP2A can boost tumor growth by inducing phosphorylation and activating these oncogenic substrate kinases ${ }^{27,28}$. ERK and JNK are members of the mitogen-activated protein kinase family. Activation of ERK has been linked to cell survival, whereas JNK participates in proliferation ${ }^{6}$. PKC is a vital messenger for the transcriptional regulation of growth factor-responsive MMP genes, which promotes cancer cell invasion ${ }^{29}$. The NF- $\kappa$ B pathway is involved in the regulation of multiple genes participating in angiogenesis of pancreatic cancer ${ }^{13}$. In this study, inhibitors of these kinase pathways markedly attenuated the cantharidininduced remodeling of the proangiogenic gene expression profiles, suggesting that all these pathways contribute to the provascularization effect of cantharidin. Notably, among the 25 investigated proangiogenic genes, the PKC inhibitor and NF- $\mathrm{kB}$ pathway inhibitor were the most effective reagents, attenuating the upregulation of 20 and 18 genes, respectively. This suggested that the PKC and NF- $\mathrm{KB}$ pathways could be the most relevant pathways participating in the proangiogenic effect of cantharidin.

TAM is a prototypical drug targeting estrogen receptor. Studies have revealed that TAM is a PKC inhibitor and can enhance the anti-pancreatic cancer effect of cantharidin by inhibiting the PKC signaling pathway in vitro ${ }^{11}$. In view of the significant role of PKC pathway in promoting angiogenesis of cantharidin, we evaluated whether TAM, as a PKC inhibitor, could antagonize the tumor-promoting effect of cantharidin. Ginsenoside Rg3, the predominant active constituent of ginseng, is a commonly used Chinese medicinal herb that inhibits biological activity of endothelial progenitor cells ${ }^{16,17}$. Furthermore, $\operatorname{Rg} 3$ has been reported to repress vascularization ${ }^{18}$. Therefore, we confirmed whether Rg3, as an antiangiogenic agent, could be used to antagonize the growth-promoting effect of cantharidin. As expected, cantharidin successfully fulfilled its antitumor potential in vivo in the presence of TAM or Rg3, presenting additive antitumor effects.

Clinical practices have proved that antiangiogenic therapy is an important and promising method for the treatment of cancer patients ${ }^{30}$. Researchers have made much effort to the direct discovery of new antiangiogenic drugs. Bevacizumab, Apatinib, and Endostar are all welldeveloped antiangiogenic agents that target VEGF, VEGFR-2, and endostatin, respectively, and have achieved considerable curative effect in patients with tumors ${ }^{31-33}$. Therefore, we evaluated the combination of cantharidin and these specific antiangiogenic therapeutics and found that these regimens presented remarkable additive antitumor effects.

Derivatives of cantharidin, such as SCAN and NCTD, have also been demonstrated to exert remarkable antitumor activity in vitro $9,34,35$. Consistent with the results for cantharidin, both SCAN and NCTD promoted tumor growth. In addition, the combination of SCAN or NCTD with bevacizumab inhibited the growth of pancreatic cancer subcutaneous xenografts dramatically.

Many reagents can repress cancer cell proliferation in vitro; however, in in vivo studies, the tumor microenvironment should be taken into consideration. Our present study revealed an unfavorable proangiogenic side effect of cantharidin via targeting the tumor angiogenic microenvironment in vivo. Antiangiogenic therapeutics or inhibitors of proangiogenic kinase pathways could antagonize the growth-promoting effect of cantharidin and present additive antitumor effects, exhibiting adequate efficacy. Therefore, rigorous preclinical investigations and clinical trials should be performed for any pharmaceuticals, even those derived from traditional 


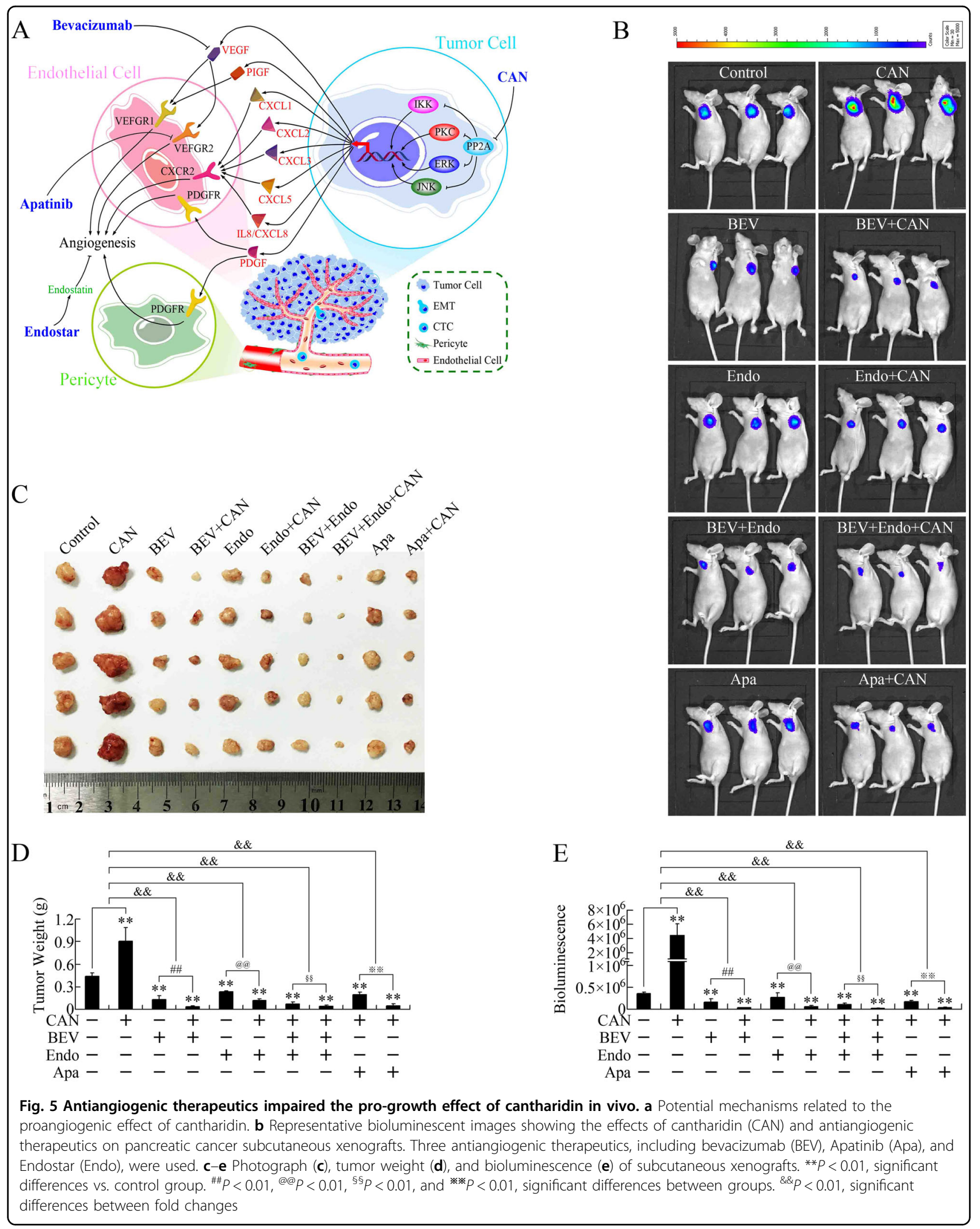




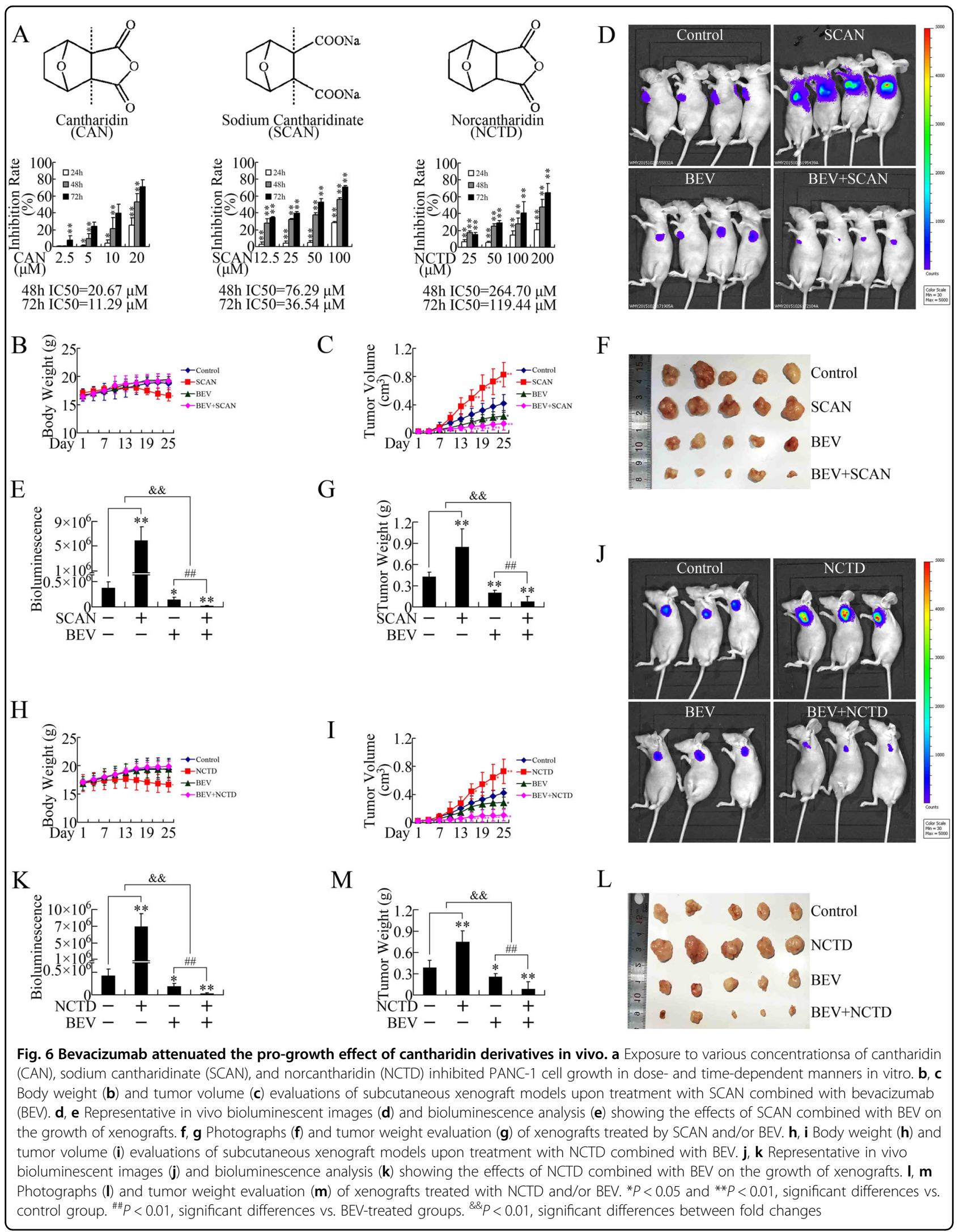


medicine. We highly recommend that clinical applications of cantharidin and its derivatives should be performed on the premise of antivascularization therapy.

\section{Materials and methods \\ Cell line and cultures}

The human pancreatic cancer cell line PANC-1, the colorectal cancer cell line LoVo, and the lung cancer cell line NCI-H292 were all purchased from the American Type Culture Collection (ATCC; Manassas, VA, USA). PANC-1 and LoVo cells were maintained in Dulbecco's modified medium (Gibco, Grand Island, NY, USA). NCIH292 cells were maintained in Roswell Park Memorial Institute-1640 medium (Gibco). In all, 10\% fetal calf serum (Gibco), $100 \mathrm{U} / \mathrm{mL}$ penicillin, and $100 \mathrm{mg} / \mathrm{mL}$ streptomycin were added to the medium. The atmosphere is humidified at $37{ }^{\circ} \mathrm{C}$ in a $5 \% \mathrm{CO}_{2}$ incubator. Cells were tested for mycoplasma contamination and passaged every 2-3 days.

\section{Reagents}

Cantharidin, OA, TAM, PD98059, SP600125, and GF109203X were purchased from Enzo Life Science International, Inc. (Plymouth Meeting, PA, USA). NCTD and EF-24 were purchased from Sigma (St. Louis, MO, USA). SCAN was purchased from Jinqiao Pharmaceutical Co. Ltd (Guizhou, China). Ginsenoside Rg3 was purchased from Shanghai Jinsui Bio-Technology Co. Ltd (Shanghai, China). Bevacizumab was purchased from Roche Pharma Co. Ltd (Basel, Switzerland). Endostar was purchased from Simcere-Medgenn Bio-pharmaceutical Co. Ltd (Shandong, China). Apatinib was purchased from Hengrui Medicine Co. Ltd (Jiangsu, China).

\section{Nude mouse tumor xenograft model and treatments}

Four-week-old female BALB/c athymic nude mice were purchased from Shanghai SLAC Laboratory Animal Co. Ltd (Shanghai, China). According to the Soochow University Institutional Animal Care and Treatment Committee, all mice were given 1 week to adapt to the new environment and received humane care throughout our experiments.

For subcutaneous xenograft model, about $1 \times 10^{7}$ cells in Matrigel (Becton Dickinson, Bedford, MA, USA) were injected together into the left side of the mice. Tumor volume was measured and recorded each day. For orthotopic pancreatic xenograft model, about $0.5 \times 10^{7}$ cells stably expressing firefly luciferase in Matrigel were injected into the body tail of the pancreas of nude mice. After sample size estimation, all mice were grouped randomly. Investigators were blinded to the group allocation during the experiments.

Treatments were conducted according to the following methods: Cantharidin, SCAN, and NCTD, dissolved in dimethyl sulfoxide (DMSO), were administered every 3 days by intraperitoneal injection at doses of 1,20 , and $1.5 \mathrm{mg} / \mathrm{kg}$, respectively. Bevacizumab $(50 \mathrm{mg} / \mathrm{kg}$ every 3 days) and Endostar (50 mg/kg per day) were administered via the caudal vein. Apatinib, TAM, and Ginsenoside Rg3 were dissolved in DMSO and administered daily by intragastric administration at doses of 10, 0.5, and $20 \mathrm{mg} / \mathrm{kg}$, respectively. For living imaging assay, the mice were anesthetized and then given $1.5 \mathrm{mg}$ D-Luciferin of $100 \mu \mathrm{L}$ phosphate-buffered serum (PBS) by intraperitoneal injection. Bioluminescence was imaged using an IVIS Lumina II apparatus (PerkinElmer, Waltham, MA, USA) or a Kodak In-Vivo Imaging System FX Pro (Rochester, NY, USA). When the experiments were terminated, the tumors were stripped and formalin-fixed for immunohistochemical assays.

\section{Immunohistochemistry}

All resected tumor samples were fixed in 10\% formalin and then embedded in paraffin by conventional treatment. Slices were cut into 4- $\mu \mathrm{m}$ thickness, heated for $30 \mathrm{~min}$ at $60{ }^{\circ} \mathrm{C}$, and then dewaxed and hydrated. The slides were microwave treated for $5 \mathrm{~min}$ with an antigen repair buffer. After supplementing the buffer, microwave $5 \mathrm{~min}$ to heat the slides once again, then cool for $20 \mathrm{~min}$. After blocking the endogenous peroxidase activity by adding $3 \% \mathrm{H}_{2} \mathrm{O}_{2}$, the sections were incubated with $10 \%$ bovine serum albumin for $1 \mathrm{~h}$ to block non-specific binding. Rabbit antiCD34 antibodies (ab81289; Abcam, London, UK) was used for immunohistochemical staining. A SuperPicture Polymer Detection Kit (No. 87-8963; Invitrogen, Carlsbad, CA, USA) was used to visualize the binding complex after incubating with the corresponding second antibody.

\section{Angiogenesis vascularity evaluation}

MVD was defined as the number of blood vessels obtained by counting each field of view at the region of high vessel density. First, vascular endothelial cells were stained with anti-CD34 antibodies. The positive expression sites were brown or brownish yellow. The sections were placed under a low power microscope to determine the region of the highest staining density, defined as the "hot spot" area, and then placed under a 400-fold microscope to count the microvessels. Individual or clustered endothelial cells were counted. There were no scores for blood vessels in the peritumoral and necrotic areas. Cells that were unclear or fuzzy stained are not counted. A vascular lumen was not an essential structure for microvessel counts. The branching structure was calculated as one, except that vascular continuity was interrupted, in which case the branch structure was calculated as two different blood vessels. MVD was calculated as the average valve of blood vessel contours counted per $\mathrm{mm}^{2}$ from at least three hot spots. 
Table 1 Primers

\begin{tabular}{|c|c|c|c|}
\hline Genes & Sense $\left(5^{\prime}-3^{\prime}\right)$ & Antisense $\left(5^{\prime}-3^{\prime}\right)$ & Product size (bp) \\
\hline B2M & TCAAGAAGGTGGTGAAGCAG & AAGGTGGAGGAGTGGGTGTC & 112 \\
\hline VEGFA & CACCCACCCACATACATACATTT & CCTCCCAACTCAAGTCCACAG & 170 \\
\hline PGF/PIGF-2 & AAGGGAGCTGCTGTCTGCG & CTTGCGGAGTCAGGAGCCCGTAGGT & 192 \\
\hline PDGFA & ATTGTAGCACTCGGTGAAGCA & TCTGGAGTCGTTCCCAAAGC & 247 \\
\hline CXCL1 & CACCCCAAGAACATCCAAAGT & CCTTCAGGAACAGCCACCA & 210 \\
\hline CXCL2 & GCTTATTGGTGGCTGTTCCTG & ACACATTAGGCGCAATCCAG & 101 \\
\hline CXCL3 & GCCCAAACCGAAGTCATAGC & GAACCCTCGTAAGAAATAGTCAAAC & 271 \\
\hline CXCL5 & ACAGTGCCCTACGGTGGAAGT & CTCATCAAAGCAGGGAGTTCATA & 266 \\
\hline IL-8/CXCL8 & TGGATGGGTTCAGAGGCAC & GCAGGGCAGAAGGAATGGT & 147 \\
\hline ILla & TGACGACGCACTTGTAGCCAC & GCCAATGAAATGACTCCCTCT & 111 \\
\hline IL10 & TGGTGAAGGAGGATCGCTAGA & CCTTGATGTCTGGGTCTTGGTT & 204 \\
\hline IL12A & TATGATGGCCCTGTGCCTTAG & CTATCAATAGTCACTGCCCGAAT & 279 \\
\hline FIGF & CATCCCATCGGTCCACTAGGT & CAGCCACCACATCGGAACA & 190 \\
\hline GM-CSF & ACACTGCTGCTGAGATGAATGA & AAAGGTGATAATCTGGGTTGCA & 218 \\
\hline CSF3 & CCTTCGCCTCTGCTITCCA & CGTTCTGCTCTTCCCTGTCTIT & 199 \\
\hline COL18A1 & TCAGACCACGGCTCGATTTC & CTCAGCTCCCATTGCCTCA & 154 \\
\hline EFNB2 & GACGTCCAGAACTAGAAGCTGG & CACCAGCGTGATGATGATGACG & 197 \\
\hline SPHK1 & GAAGGGTGAGAAGGTGGAGG & TTATTGGATTGGTTCGTGGG & 147 \\
\hline TIE1 & CCAAGTACGTTGTGGAGGTGC & ACGGATGATGGTGCTTGTCTC & 95 \\
\hline BTG1 & CGGGTTACCGTTGTATTCGC & TTCGGCTGTCTACCATTTGC & 246 \\
\hline CD55 & GCTAAATTCTGCATCCCTCAAAC & TGTAACCTGGACGGCACTCAT & 92 \\
\hline KDR & CCCAATAATCAGAGTGGCAGTG & CATAGACATAAATGACCGAGGCC & 163 \\
\hline KITLG & TGTTGGATAAGCGAGATGGTAGT & TTCACGCACTCCACAAGGTC & 146 \\
\hline PLAUR/UPAR & GCCGGGCTGTCACCTATT & CCACATCCAGGCACTGTTCTTC & 132 \\
\hline $\mathrm{RHOB}$ & GCATCCAAGCCTACGACTACCT & CGGCCCTCATAGCACCTT & 149 \\
\hline RUNX1 & TGCCTCAGTGGAGACAAGTGG & GTCTCAGCCTGGTGAAAGCA & 107 \\
\hline
\end{tabular}

\section{Milliplex assay}

Secretion levels of IL-6, IL-8, TNF- $\alpha$, and VEGF were detected using a multiplex biometric immunoassay (Cat. No. HCYTOMAG-60K; Millipore). Luminex Technology (Bio-Plex Workstation; Bio-Rad Laboratories, Hercules, CA, USA) was used to calculate the mean fluorescence intensity. Milliplex ${ }^{\circledR}$ Analyst 5.1 (Bio-Rad Laboratories) was applied for data analysis.

\section{Angiogenesis antibody array}

Proteins were biotinylated in the samples and the superfluous biotin was removed by spin-filter. After dialyzing by PBS, biotinylated proteins were added to RayBio Human Angiogenesis Antibody Array membranes (RayBiotech, Norcross, GA, USA). By incubation with horseradish peroxidase-streptavidin, the captured biotinylated proteins could be measured. Then a chemiluminescence imaging system (RayBiotech) was applied to analyze the results. The internal control signals of each protein array chip were used for standardization. Quality control was performed by removing proteins with a signal intensity $<50$. A $t$ test was used for the difference analysis, and fold changes $\geq 1.5$ times were considered significant. Standardization was performed with internal control signals on each protein chip to remove proteins that were 50 times lower than the original signal intensity.

\section{Real-time PCR}

Total RNA was first extracted by TRIzol reagent (Invitrogen) following its protocol. After quantification by spectrophotometry, reverse transcription was carried out in a system of $20 \mu \mathrm{L}$ with $1 \mu \mathrm{g}$ total RNA using a 
PrimeScript RT Kit (Takara, Otsu, Shiga, Japan) following the manufacturer's protocol. Aliquots of cDNA were placed on a LightCycler 96 Real-time Quantitative PCR Detection system (Roche, Indianapolis, IN, USA) for PCR amplification. The reaction system was $25 \mu \mathrm{L}$, which consisted of the cDNA, forward and reverse primers, and SYBR Green PCR master mix (Roche). Three sets of duplicate wells were set for each sample, and the reaction was terminated after 40 cycles. The $\mathrm{Ct}$ value of each system was recorded. $B 2 M$ gene was used as an internal standard. The relative transcription levels of mRNA were calculated by $2^{-\Delta C T}$. Specific primers used in this study are listed in Table 1 .

\section{MTT assay}

MTT was performed to assess the ability of cell growth. Cells in logarithmic growth phase were adjusted to an appropriate cell density and seeded into 24-well culture plates. Twenty-four hours later, the cells were grouped and administered treatment according to the experimental requirements. After treatment, MTT and DMSO were added separately according to the protocol described in our previous study ${ }^{6}$. The absorbance value was measured at $490 \mathrm{~nm}$ by a microplate enzyme-linked immunosorbent assay reader (Bio-Rad Laboratories). Inhibition rate $=[($ mean control absorbance - mean experimental absorbance)/mean control absorbance] $\times 100(\%)$.

\section{RNA-seq analysis}

First, total RNA was extracted using TRIzol reagent (Invitrogen) following its protocol. An RNeasy Mini Column (Qiagen, CA, USA) was then used to purify the extracted RNA. After quality evaluation, total RNA was sequenced and analyzed for mRNA expression profile (Novel Bioinformatics, Shanghai, China). We used FASTQC to assess the original sequencing data and TopHat for RNA-seq alignment ${ }^{36}$. To identify the possible splicing junctions, MAQ was used to assemble the mapped reads. Besides, the unmapped reads also could define the splice junctions using the seed extension procedure. We performed the Limma algorithm to filter the genes after the analysis of significance and false discovery rate (FDR) according to the criteria: (i) fold change $>1.5$ or $<0.67$, (ii) FDR $<0.05^{36}$. Data has been uploaded to GEO database, the accession code of which is GSE114040.

\section{Statistical analysis}

All experiments were conducted at least three times. The results were calculated as mean \pm standard deviation. An unpaired Student's $t$ test was used for statistical analysis. An $F$-test to compare variances was performed, if necessary. $P<0.05$ was considered statistically significant.

\section{Acknowledgements}

This work was supported by the National Natural Science Foundation of China (grant numbers 81472296, 81602091, 81402176, 81501970, 81272542, 81200369), the Six Major Talent Peak Project of Jiangsu Province (grant number 2015-WSN-022), the Project of Invigorating Health Care through Science, Technology and Education, Jiangsu Provincial Medical Youth Talent (grant number QNRC2016709), the Project of Jiangsu Provincial Commission of Health and Family Planning (grant number H201518), and the Science and Education for Health Foundation of Suzhou for Youth (grant number kjxw2015003).

\section{Author details \\ 'Department of Oncology, the First Affiliated Hospital of Soochow University, 215006 Suzhou, China. ${ }^{2}$ Department of Emergency, the First Affiliated Hospital of Soochow University, 215006 Suzhou, China. ${ }^{3}$ Department of Oncology, the First Affiliated Hospital of Zhejiang Chinese Medicine University, 310006 Hangzhou, China. ${ }^{4}$ Department of Radio-Oncology, Nanjing Medical University Affiliated Suzhou Hospital, 215001 Suzhou, China. ${ }^{5}$ Department of Hematology, the First Affiliated Hospital of Soochow University, 215006 Suzhou, China. ${ }^{6}$ PREMED Key Laboratory for Precision Medicine, Soochow University, 215021 Suzhou, China. ${ }^{7}$ Department of General Surgery, the First Affiliated Hospital of Soochow University, 215006 Suzhou, China. ${ }^{8}$ Comprehensive Cancer Center, Suzhou Xiangcheng People's Hospital, 215000 Suzhou, China}

Conflict of interest

The authors declare that they have no conflict of interest.

\section{Publisher's note}

Springer Nature remains neutral with regard to jurisdictional claims in published maps and institutional affiliations.

Supplementary Information accompanies this paper at (https://doi.org/ 10.1038/s41389-018-0102-2).

Received: 14 April 2018 Revised: 14 July 2018 Accepted: 9 November 2018 Published online: 26 November 2018

\section{References}

1. Kamisawa, T., Wood, L. D., Itoi, T. \& Takaori, K. Pancreatic cancer. Lancet 388, 73-85 (2016).

2. von Ahrens, D., Bhagat, T. D., Nagrath, D., Maitra, A. \& Verma, A. The role of stromal cancer-associated fibroblasts in pancreatic cancer. J. Hematol. Oncol. 10, 76 (2017)

3. Siegel, R. L., Miller, K. D. \& Jemal, A. Cancer Statistics, 2017. CA Cancer J. Clin. 67, 7-30 (2017).

4. Li, W. et al. Cantharidin, a potent and selective PP2A inhibitor, induces an oxidative stress-independent growth inhibition of pancreatic cancer cells through G2/M cell-cycle arrest and apoptosis. Cancer Sci. 101, 1226-1233 (2010).

5. Gong, F. R. et al. PP2A inhibitors arrest G2/M transition through JNK/Sp1dependent down-regulation of CDK1 and autophagy-dependent up-regulation of p21. Oncotarget 6, 18469-18483 (2015).

6. Li, W. et al. Growth of the pancreatic cancer cell line PANC-1 is inhibited by protein phosphatase $2 \mathrm{~A}$ inhibitors through overactivation of the c-Jun $\mathrm{N}$ terminal kinase pathway. Eur. J. Cancer 47, 2654-2664 (2011).

7. Li, W. et al. PP2A inhibitors induce apoptosis in pancreatic cancer cell line PANC-1 through persistent phosphorylation of IKKalpha and sustained activation of the NF-kappaB pathway. Cancer Lett. 304, 117-127 (2011).

8. Shen, M. et al. Cantharidin represses invasion of pancreatic cancer cells through accelerated degradation of MMP2 mRNA. Sci. Rep. 5, 11836 (2015).

9. Wang, W. J. et al. Cantharidin and norcantharidin impair stemness of pancreatic cancer cells by repressing the beta-catenin pathway and strengthen the cytotoxicity of gemcitabine and erlotinib. Int. J. Oncol. 47, 1912-1922 (2015).

10. Wu, M. Y. et al. PP2A inhibitors suppress migration and growth of PANC-1 pancreatic cancer cells through inhibition on the Wnt/beta-catenin pathway 
by phosphorylation and degradation of beta-catenin. Oncol. Rep. 32, 513-522 (2014).

11. Xie, X. et al. Tamoxifen enhances the anticancer effect of cantharidin and norcantharidin in pancreatic cancer cell lines through inhibition of the protein kinase C signaling pathway. Oncol. Lett. 9, 837-844 (2015).

12. Aronis, K. N., Chamberland, J. P. \& Mantzoros, C. S. GLP-1 promotes angiogenesis in human endothelial cells in a dose-dependent manner, through the Akt, Src and PKC pathways. Metabolism 62, 1279-1286 (2013).

13. Xiong, H. Q. et al. NF-kappaB activity blockade impairs the angiogenic potential of human pancreatic cancer cells. Int. J. Cancer 108, 181-188 (2004).

14. Yang, X. J. et al. TGF-beta1 enhances tumor-induced angiogenesis via JNK pathway and macrophage infiltration in an improved zebrafish embryo/ xenograft glioma model. Int. Immunopharmacol. 15, 191-198 (2013).

15. Zhao, W. et al. RERG suppresses cell proliferation, migration and angiogenesis through ERK/NF-kappaB signaling pathway in nasopharyngeal carcinoma. J. Exp. Clin. Cancer Res. 36, 88 (2017).

16. Kim, J. W. et al. Ginsenoside Rg3 attenuates tumor angiogenesis via inhibiting bioactivities of endothelial progenitor cells. Cancer Biol. Ther. 13, 504-515 (2012).

17. Kim, J. W. et al. Ginsenoside Rg3 inhibits endothelial progenitor cell differentiation through attenuation of VEGF-dependent Akt/eNOS signaling. Phytother. Res. 26, 1286-1293 (2012).

18. Chen, Q. J., Zhang, M. Z. \& Wang, L. X. Gensenoside Rg3 inhibits hypoxiainduced VEGF expression in human cancer cells. Cell. Physiol. Biochem. 26, 849-858 (2010).

19. Yue, P. Y. et al. The angiosuppressive effects of 20(R)- ginsenoside Rg3. Biochem. Pharmacol. 72, 437-445 (2006).

20. Wang, W. Q. et al. Intratumoral alpha-SMA enhances the prognostic potency of CD34 associated with maintenance of microvessel integrity in hepatocellular carcinoma and pancreatic cancer. PLOS ONE 8, e71189 (2013).

21. Baeriswyl, V. \& Christofori, G. The angiogenic switch in carcinogenesis. Semin. Cancer Biol. 19, 329-337 (2009).

22. Volpert, O. V., Dameron, K. M. \& Bouck, N. Sequential development of an angiogenic phenotype by human fibroblasts progressing to tumorigenicity. Oncogene 14, 1495-1502 (1997).

23. Burmeister, $K$. et al. Vascular endothelial growth factor A amplification in colorectal cancer is associated with reduced M1 and M2 macrophages and diminished PD-1-expressing lymphocytes. PLOS ONE 12, e0175563 (2017).

24. Xie, K. Interleukin-8 and human cancer biology. Cytokine Growth Factor Rev. 12 375-391 (2001).

25. Benoy, I. H. et al. Increased serum interleukin-8 in patients with early and metastatic breast cancer correlates with early dissemination and survival. Clin. Cancer Res. 10, 7157-7162 (2004)

26. Ren, Y. et al. Interleukin-8 serum levels in patients with hepatocellular carcinoma: correlations with clinicopathological features and prognosis. Clin. Cancer Res. 9, 5996-6001 (2003).

27. Janssens, V., Goris, J. \& Van Hoof, C. PP2A: the expected tumor suppressor. Curr. Opin. Genet. Dev. 15, 34-41 (2005).

28. Millward, T. A., Zolnierowicz, S. \& Hemmings, B. A. Regulation of protein kinase cascades by protein phosphatase 2A. Trends Biochem. Sci. 24, 186-191 (1999).

29. Chakraborti, S. Mandal, M. Das, S. Mandal, A. \& Chakraborti, T. Regulation of matrix metalloproteinases: an overview. Mol. Cell. Biochem. 253, 269-285 (2003).

30. Fan, F., Schimming, A., Jaeger, D. \& Podar, K. Targeting the tumor microenvironment: focus on angiogenesis. J. Oncol. 2012, 281261 (2012).

31. Gasparini, G., Longo, R., Fanelli, M. \& Teicher, B. A. Combination of antiangiogenic therapy with other anticancer therapies: results, challenges, and open questions. J. Clin. Oncol. 23, 1295-1311 (2005).

32. Xing, P. et al. Recombined humanized endostatin (Endostar) combined with chemotherapy for advanced bone and soft tissue sarcomas in stage IV. Oncotarget 8, 36716-36727 (2017).

33. Zhang, M. et al. Concurrent apatinib and local radiation therapy for advanced gastric cancer: A case report and review of the literature. Medicine (Baltimore) 96, e6241 (2017).

34. Shou, L. M. et al. Cantharidin and norcantharidin inhibit the ability of MCF-7 cells to adhere to platelets via protein kinase $C$ pathway-dependent downregulation of alpha2 integrin. Oncol. Rep. 30, 1059-1066 (2013).

35. Tao, R. et al. Role of S100A3 in human hepatocellular carcinoma and the anticancer effect of sodium cantharidinate. Exp. Ther. Med. 13, 2812-2818 (2017).

36. Shen, Y. et al. Identifying microRNA-mRNA regulatory network in gemcitabineresistant cells derived from human pancreatic cancer cells. Tumour Biol. 36, 4525-4534 (2015) 